\title{
NON-OSCILLATING PALEY-WIENER FUNCTIONS
}

\author{
By
}

\author{
I. V. OSTROVSKII AND A. ULANOVSKII
}

\begin{abstract}
A non-oscillating Paley-Wiener function is a real entire function $f$ of exponential type belonging to $L_{2}(\mathbf{R})$ and such that each derivative $f^{(n)}, n=$ $0,1,2, \ldots$, has only a finite number of real zeros. It is established that the class of such functions is non-empty and contains functions of arbitrarily fast decay on $\mathbf{R}$ allowed by the convergence of the logarithmic integral. It is shown that the Fourier transform of a non-oscillating Paley-Wiener function must be infinitely differentiable outside the origin. We also give close to best possible asymptotic (as $n \rightarrow \infty$ ) estimates of the number of real zeros of the $n$-th derivative of a function $f$ of the class and the size of the smallest interval containing these zeros.
\end{abstract}

\section{Introduction}

A Paley-Wiener function (PW-function) is an entire function $f$ of exponential type such that $f \in L_{2}(\mathbf{R})$. By the Paley-Wiener theorem, the class of all PWfunctions coincides with the class of functions $f$ admitting the representation

$$
f(z)=\int_{-\infty}^{\infty} e^{-i z s} F(s) d s
$$

where $F$ (called the spectral function of $f$ ) is an $L_{2}$-function with bounded support.

We say that a PW-function $f$ is real if $f(\mathbf{R}) \subset \mathbf{R}$. Oscillatory properties of real PW-functions have been the subject of investigation of a number of works (see, for example, [1], [3] and [8]). J. R. Higgins [3] (p. 77) has constructed a sequence of real PW-functions $s_{n}$ with the following property. Each $s_{n}$ has a derivative of order $2 n$ with infinitely many real zeros, but each derivative of order less than $2 n$ has only a finite number of real zeros. W. J. Walker [8] (p. 1254) and J. R. Higgins [3] (p. 72) posed the question: Does a real PW-function $f$ have for some order $n$ a derivative $f^{(n)}$ which has infinitely many real zeros? For some classes of real PW-functions, a positive answer was obtained by W. J. Walker [8] and J. Clunie et al. [1]. In the latter work, it is mentioned (p. 167) that the answer to this question is still unknown. 
In this paper, we answer this question in the negative. Let us call a PW-function $f$ non-oscillating if it is real and each derivative $f^{(n)}$ has only a finite number of real zeros. Simple examples of such functions are given in the following result.

Theorem 1. (i) Suppose a real function $p \in C^{\infty}(\mathbf{R})$ has bounded support and satisfies $p(0) \neq 0$. Then the function

$$
f_{1}(x):=\int_{0}^{\infty} p(s) \sin x s d s
$$

is a non-oscillating PW-function.

(ii) Suppose $p \in C^{\infty}(\mathbf{R})$ has a bounded support, $p(-s)=\bar{p}(s)$, for all real $s$, and $\int_{-\infty}^{\infty} p(s) d s \neq 0$. Then the function

$$
f_{2}(x):=\frac{1}{x} \int_{-\infty}^{\infty}\left(1-e^{i x s}\right) p(s) d s
$$

is a non-oscillating $P W$-function.

Proof. Since $p$ has compact support, the function $f_{1}$ is a real PW-function. Choose any integer $n \geq 1$. The statement (i) can be proved simply by integrating by parts $2 n$ times:

$$
\begin{gathered}
f_{1}^{(2 n-1)}(x)=(-1)^{n-1} \int_{0}^{\infty} s^{2 n-1} p(s) \cos x s d s \\
=\left[\frac{\cos x s}{x^{2 n}}\left(s^{2 n-1} p(s)\right)^{(2 n-1)}\right]_{0}^{\infty}-\frac{1}{x^{2 n}} \int_{0}^{\infty}\left(s^{2 n-1} p(s)\right)^{(2 n)} \cos x s d s .
\end{gathered}
$$

Since the function $\left(s^{2 n-1} p(s)\right)^{(2 n)}$ is continuous and has bounded support, the last integral tends to zero as $|x| \rightarrow \infty$. We conclude that

$$
\left|f_{1}^{(2 n-1)}(x)\right|=(2 n-1) ! \frac{|p(0)|}{x^{2 n}}(1+o(1)), \quad|x| \rightarrow \infty, \quad n=1,2, \ldots
$$

This shows that for any $n \geq 1$ the function $f_{1}^{(2 n-1)}$ can have only a finite number of real zeros. Hence, by Rolle's theorem, any derivative $f_{1}^{(n)}, n=1,2, \ldots$ has only a finite number of real zeros.

(ii) It is clear that $f_{2}$ is a real PW-function. Observe that

$$
f_{2}^{(n)}(x)=\frac{(-1)^{n} n !}{x^{n+1}} \int_{-\infty}^{\infty} p(s) d s-\int_{-\infty}^{\infty} p(s)\left(\frac{e^{i x s}}{x}\right)^{(n)} d s
$$


Since the the function $p$ is infinitely differentiable on the real line, for any natural number $k$, the second integral in the right hand side is $o\left(|x|^{-k}\right)$ as $x \rightarrow \infty$. This shows that

$$
\left|f_{2}^{(n)}(x)\right|=\frac{n !}{|x|^{n+1}}\left|\int_{-\infty}^{\infty} p(s) d s\right|(1+o(1)), \quad|x| \rightarrow \infty, \quad n=1,2, \ldots,
$$

so that every derivative $f_{2}^{(n)}$ can have only a finite number of real zeros.

The functions $f_{1}$ and $f_{2}$ in Theorem 1 tend to zero like $|x|^{-1}$, and their $n$-th derivatives tend to zero like $|x|^{-n-1}$ as $|x| \rightarrow \infty, x \in \mathbf{R}$. Since the $n$-th derivative of a non-oscillating PW-function is also a non-oscillating PW-function, for any $n=1,2, \ldots$ there exist non-oscillating PW-functions $f$ which satisfy $|f(x)|=$ $O\left(|x|^{-n}\right)$ as $|x| \rightarrow \infty$. Below, we show that non-oscillating PW-functions $f$ can have arbitrarily fast decay provided that the logarithmic integral converges (which is true for every PW-function):

$$
\int_{-\infty}^{\infty} \frac{|\log | f(x)||}{1+x^{2}} d x<\infty
$$

For instance, Theorem 4 below implies that for any $\rho \in(0,1)$ and any $\sigma>0$, there exists a real non-oscillating PW-function $f$ of type $\sigma$ such that

$$
|x|^{\rho} \log \frac{1}{|f(x)|}
$$

is bounded from below and above by positive constants for all sufficiently large $|x|, x \in \mathbf{R}$.

There remains an open question related to the slowest possible rate of decay of a real non-oscillating $P W$-function on $\mathbf{R}$. For example, we do not know whether there exist non-oscillating PW-functions decreasing on $\mathbf{R}$ like $|x|^{-\alpha}$ with $1 / 2<\alpha<1$.

Observe that the spectral function of the function $f_{1}$ in Theorem 1 is $(i / 2) p(|s|)$ sign $s, s \in \mathbf{R}$. This function is infinitely differentiable outside the origin. One can verify that this is also true for the spectral function of $f_{2}$. Our next result shows that the spectral function of any non-oscillating PW-function has this property.

Theorem 2. Let $f$ be a non-oscillating $P W$-function. Then its spectral function belongs to $C^{\infty}(\mathbf{R} \backslash\{0\})$.

Let us denote by $r(n, f)$ the maximum of the moduli of real zeros of the $n$-th derivative of a non-oscillating PW-function $f$ and by $p(n, f)$ the number of its real zeros. Rolle's theorem implies that both $r(n, f)$ and $p(n, f)$ are strictly increasing functions of $n$ and, moreover, $p(n, f) \geq n$. It seems natural to ask how fast $r(n, f)$ and $p(n, f)$ may grow as $n \rightarrow \infty$. 
Theorem 3. Let $f$ be a non-oscillating $P W$-function. Then

$$
\log r(n, f) \geq(1+o(1)) \log n, \quad n \rightarrow \infty .
$$

The inequality in (1) is sharp in the sense that there are functions $f$ for which the inequality can be replaced by equality. We deduce Theorem 3 from the more general Theorem 5 below.

Observe that inequality (1) also remains valid for $p(n, f)$. This follows from the trivial inequality $p(n, f) \geq n$ and Corollary 1 below. The problem of finding more precise bounds and any upper bound for $r(n, f)$ and $p(n, f)$ remains open.

\section{Rate of decay and real zeros of a non-oscillating PW-function}

We assume familiarity with the notion of a proximate order ([2], Ch. 2, $\S 2$; [4], Ch. 1, sec. 12). Recall that a proximate order is a continuously differentiable positive function $\rho(r)$ on $[0, \infty)$ satisfying the conditions

(i) $\exists \lim _{r \rightarrow \infty} \rho(r)=: \rho \geq 0$;

(ii) $\lim _{r \rightarrow \infty}\left(\rho^{\prime}(r) / \rho(r)\right) r \log r=0$.

Put

$$
V(r):=r^{\rho(r)}
$$

The simplest example of a proximate order is $\rho(r) \equiv \rho>0$. In this case, $V(r)=r^{\rho}$; and the considerations below would be significantly simpler and independent of results of [2] and [4] related to proximate orders.

Condition (ii) implies that the function $V$ is strictly increasing for sufficiently large $r$. Evidently, it is possible to change $\rho(r)$ on a large interval $[0, R]$ in such a way that $V$ becomes strictly increasing on $[0, \infty)$ and $V(0)=0$. We shall assume that this change has been made.

The following result relates to the rate of decay on $\mathbf{R}$ of non-oscillating PW-functions.

Theorem 4. Let $\rho(r)$ be a proximate order such that

$$
\lim _{r \rightarrow \infty} \rho(r)=\rho>0, \quad \text { and } \quad \int_{1}^{\infty} \frac{V(r)}{r^{2}} d r<\infty .
$$

There exists a real non-oscillating $P W$-function $f$ such that

$$
C_{1}<\frac{1}{V(|x|)} \log \frac{1}{|f(x)|}<C_{2}, \quad|x|>r_{0}, x \in \mathbf{R},
$$

where $r_{0}, C_{1}$ and $C_{2}$ are positive constants. 
Let $f$ be a non-oscillating PW-function and $r(n, f)$ the maximum of the moduli of real zeros of $f^{(n)}$. Our next result estimates the rate of the increase of $r(n, f)$.

Theorem 5. (i) For any proximate order $\rho(r)$ satisfying (2), there exists a non-oscillating $P W$-function $f$ such that

$$
r(n, f) \leq v(C n \log n), \quad n=0,1,2, \ldots,
$$

where $v$ is the inverse function for $V$ and $C$ is a positive constant.

(ii) For any non-oscillating $P W$-function $f$ of type 1 ,

$$
r(n, f) \geq \frac{\pi}{2 e} n(1+o(1)), \quad n \rightarrow \infty .
$$

We derive from Theorem 5 (i) the following fact, related to the possible growth of the number $p(n, f)$ of real zeros of the $n$-th derivative of a non-oscillating PW function $f$.

Corollary 1. For any proximate order $\rho(r)$ satisfying (2), there exists a nonoscillating $P W$-function $f$ such that

$$
p(n, f) \leq v(C n \log n), \quad n=0,1,2, \ldots,
$$

where $v$ is the inverse function for $V$ and $C$ is a positive constant.

Evidently, Theorem 5 (ii) implies inequality (1). Applying Theorem 5 (i) to the proximate order $\rho(r)$ such that $V(r)=r^{\rho(r)}=r / \log ^{2}(1+r)$, we see that there exists a non-oscillating PW-function $f$ for which $r(n, f)=O\left(n \log ^{2} n\right), n \rightarrow \infty$. For this function, the inequality in (1) becomes equality. Similarly, Corollary 1 implies that there exists a non-oscillating PW-function such that $\log p(n, f)=$ $(1+o(1)) \log n, n \rightarrow \infty$.

The rest of this paper is organized as follows. Some auxiliary results are proved in the next section. Section 4 is then devoted to the proof of Theorem 4 , and Sections 5 and 6 to the proof of Theorem 5 and Corollary 1. An auxiliary result which is used in the proof of Theorem 5 is proved in Section 7. Finally, Theorem 2 is proved in Section 8.

Theorems 4 and 5 were announced in [7] with a short description of the method of proof.

\section{Auxiliary lemmas}

Lemma 1. Let $\rho_{1}(r)$ be a proximate order such that $\lim _{r \rightarrow \infty} \rho_{1}(r)=\rho_{1} \in(0,1)$ and let $V_{1}(r)=r^{\rho_{1}(r)}$. Define an increasing sequence of positive numbers $a_{k}$ as 
the solutions of the equations

$$
V_{1}(r)=k, k=1,2,3, \ldots,
$$

and set

$$
h_{1}(z)=\prod_{k=1}^{\infty}\left(1-\frac{z}{a_{k}}\right)
$$

Then the circles

$$
C_{k}(\epsilon)=\left\{z:\left|z-a_{k}\right| \leq \epsilon a_{k} / V_{1}\left(a_{k}\right)\right\}, \quad k=1,2,3, \ldots
$$

do not intersect each other provided that number $\epsilon$ is small enough. Further, the estimate

(8) $\quad \log \left|h_{1}\left(r e^{i \varphi}\right)\right|=\frac{\pi \cos \rho_{1}(\varphi-\pi)}{\sin \pi \rho_{1}} V_{1}(r)+o\left(V_{1}(r)\right), \quad r \rightarrow \infty, \quad 0 \leq \varphi \leq 2 \pi$

holds outside the union of the cicrles $C_{k}(\epsilon)$.

Proof. It follows immediately from the definition (7) and the properties of proximate order that the circles $C_{k}(\epsilon)$ do not intersect for all sufficiently small $\epsilon>0$. This means that the roots $a_{k}$ of the function $h_{1}$ form a so-called $R$-set (for the definition see [4], p. 95). The asymptotic formula (8) for $r e^{i \varphi} \notin \bigcup_{k} C_{k}(\epsilon)$ now follows from a known result on entire functions of completely regular growth ([4], p. 96, Theorem 5).

\section{Corollary 2. Set}

$$
h_{2}(z)=h_{1}\left(-z^{2}\right)=\prod_{k=1}^{\infty}\left(1+\frac{z^{2}}{a_{k}}\right) .
$$

Then $h_{2}(z)$ is positive on $\mathbf{R}$, real on $i \mathbf{R}$ and

(i)

$$
\log h_{2}(x)=\frac{\pi}{\sin \pi \rho_{1}} V_{1}\left(x^{2}\right)+o\left(V_{1}\left(x^{2}\right)\right), \quad|x| \rightarrow \infty ;
$$

(ii) on every interval $\left(\sqrt{a_{k}}, \sqrt{a_{k+1}}\right)$, there is a point $b_{k}, k=1,2, \ldots$, such that

$$
\log \left|h_{2}\left( \pm i b_{k}\right)\right|=\pi \cot \pi \rho_{1} \cdot V_{1}\left(b_{k}^{2}\right)+o\left(V_{1}\left(b_{k}^{2}\right)\right), \quad b_{k} \rightarrow \infty .
$$

Lemma 2. Let $\rho(r), \lim _{r \rightarrow \infty} \rho(r)=\rho \in(0,1]$ be a proximate order. Then there exists an entire function

$$
g(z)=\prod_{k=1}^{\infty}\left(1+\frac{z^{2}}{c_{k}^{2}}\right), \quad 0<c_{1}<c_{2}<\cdots, \quad \sum_{k} c_{k}^{-2}<\infty,
$$

which is positive on $\mathbf{R}$, real on $i \mathbf{R}$ and such that 
(i)

$$
\log g(x)=\frac{\pi}{\sin (\pi \rho / 2)} V(|x|)+o(V(|x|)), \quad|x| \rightarrow \infty
$$

(ii) every interval $\left(c_{k}, c_{k+1}\right)$ contains a point $d_{k}, k=1,2, \ldots$, such that

$$
\log \left|g\left( \pm i d_{k}\right)\right|=\pi \cot \frac{\pi \rho}{2} \cdot V\left(d_{k}\right)+o\left(V\left(d_{k}\right)\right), \quad d_{k} \rightarrow \infty .
$$

To establish this lemma, it suffices to apply Corollary 2 with $\rho_{1}=\frac{1}{2} \rho$ and set $g(z)=h_{2}(z), c_{k}=\sqrt{a_{k}}, d_{k}=b_{k}$.

Lemma 3. Suppose $\rho(r)$ is a proximate order satisfying (2). Then there exists an even real $P W$-function $h$ with real zeros such that

$$
\log |h(x)| \leq-V(|x|)+O(1), \quad|x| \rightarrow \infty, \quad x \in \mathbf{R} .
$$

Proof. This lemma is a special case of the Beurling-Malliavin multiplier theorem. We give the proof below for the reader's convenience.

Assume additionally that $\lim _{r \rightarrow \infty} \rho(r)=1$. Let $\left\{a_{k}\right\}$ be a sequence of positive numbers defined by the equations

$$
V\left(a_{k}\right)=k, \quad k=1,2, \ldots
$$

Set

$$
h_{1}(z)=\prod_{k=1}^{\infty} \frac{\sin \left(z / a_{k}\right)}{z / a_{k}} .
$$

It is easy to verify that (2) yields $\sum_{k}\left(1 / a_{k}\right)<\infty$. Hence, this infinite product converges and represents an entire function of exponential type.

Let $n(r)$ be the number of points $a_{k}$ to be found in the disc $\{z:|z| \leq r\}$. We have, for every real $x$ and integer $n$, that

$$
\left|h_{1}(x)\right| \leq \frac{1}{|x|^{n}} \prod_{k=1}^{n} a_{k} .
$$

Now set $n=n(|x|)$ and observe that

$$
\left|h_{1}(x)\right| \leq \exp \left\{-\int_{0}^{|x|} \frac{n(t)}{t} d t\right\} .
$$

It is easily seen from (2) that $n(t)$ is asymptotically equivalent to $t^{\rho(t)}$. Hence, by known properties of the proximate order ([4], p. 34), we conclude that

$$
\int_{0}^{|x|} \frac{n(t)}{t} d t=V(|x|)(1+o(1))
$$


This gives

$$
\log \left|h_{1}(x)\right| \leq-V(|x|)(1+o(1)) \leq-\frac{1}{2} V(|x|)+C .
$$

Hence the function $h(z)=h_{1}\left(z^{2}\right)$ satisfies the conclusions of the Lemma.

In the case $\lim _{r \rightarrow \infty} \rho(r)=\rho<1$, one can construct $h_{1}(z)$ by taking a constant $\rho_{1} \in(\rho, 1)$ instead of the function $\rho(r)$. Then one gets

$$
\log \left|h_{1}(x)\right| \leq-\frac{1}{p_{1}}|x|^{\rho_{1}}(1+o(1)) \leq-V(|x|)(1+o(1)),
$$

and again one can set $h(z)=h_{1}^{2}(z)$.

Lemma 4. Suppose $\rho(r)$ is a proximate order, and $q(z)$ is an entire function of completely regular growth with respect to $\rho(r)$. Assume that

(i) $q(z)$ does not vanish in $\{z:|\arg z| \leq \alpha<\pi\}$,

(ii) $\exists \lim _{x \rightarrow+\infty}(\log |q(x)|) / V(x) \neq 0$.

Then the derivative $q^{\prime}(z)$ does not vanish in $\{z:|\arg z| \leq \alpha / 3,|z|>R\}$ for all large $R$, and the asymptotic equation

$$
\begin{gathered}
\left(\frac{d}{d z}\right)^{n} \frac{1}{q(z)}=(-1)^{n}\left(\frac{q^{\prime}(z)}{q(z)}\right)^{n} \frac{1}{q(z)}\left(1+O\left(\frac{1}{V(|z|)}\right)\right), \\
z \rightarrow \infty, \quad|\arg z| \leq \alpha / 3
\end{gathered}
$$

holds for all $n=1,2, \ldots$

Proof. We require the following fact (see [2], p. 99, Theorem 5.1). Let $\rho(r)$, $\lim _{r \rightarrow \infty} \rho(r)=\rho$ be a proximate order. Then there exists a function $L(z)$ analytic in $\{z:|\arg z|<\pi\}$ such that

$$
V(r)\left(=r^{\rho(r)}\right)=r^{\rho} L(r)(1+o(1)), \quad r \rightarrow \infty
$$

where (15) holds uniformly in $\varphi \in(-\pi+\delta, \pi-\delta)$ for every $\delta \in(0, \pi)$.

Let us verify that (15) gives

$$
L^{\prime}\left(r e^{i \varphi}\right)=o\left(\frac{L(r)}{r}\right), \quad r \rightarrow \infty
$$


uniformly with respect to $\varphi \in[-\pi / 2, \pi / 2]$. Indeed, since ([2], p. 73, [4], p. 32)

$$
\lim _{r \rightarrow \infty} \frac{L(k r)}{L(r)}=1
$$

for every $k>0$, then, in view of (15),

$$
L(\zeta)=L(r)+o(L(r)), \quad r \rightarrow \infty,
$$

uniformly with respect to $\zeta$ in the sector $\{\zeta:|\arg \zeta|<2 \pi / 3, r / 2<|\zeta|<3 r / 2\}$. It now follows from the Cauchy integral formula that

$$
\begin{gathered}
L^{\prime}\left(r e^{i \varphi}\right)=\frac{1}{2 \pi i} \int_{\left|\zeta-r e^{i \varphi}\right|=r / 2} \frac{L(\zeta)}{\left(\zeta-r e^{i \varphi}\right)^{2}} d \zeta \\
=\frac{1}{2 \pi i} \int_{\left|\zeta-r e^{i \varphi}\right|=r / 2} \frac{L(\zeta)-L(r)}{\left(\zeta-r e^{i \varphi}\right)^{2}} d \zeta=o\left(\frac{L(r)}{r}\right), \quad r \rightarrow \infty,
\end{gathered}
$$

which establishes (16).

By known results in the theory of functions of completely regular growth ([4], pp. 94-95, Theorem 4), we have

$$
\log q(z)=A z^{\rho} L(r)+o(V(r)), \quad r \rightarrow \infty, z=r e^{i \varphi},|\varphi| \leq \alpha / 2,
$$

where $A$ is the limit in assumption (ii) of the lemma. This and (15) give

$$
\log q(z)=A z^{\rho} L(z)+o(V(r)), \quad r \rightarrow \infty, z=r e^{i \varphi},|\varphi| \leq \alpha / 2 .
$$

Let us now differentiate the formula. Using (16) and estimating the derivative of the remainder term with the help of the Cauchy integral, we get

$$
\begin{gathered}
\frac{q^{\prime}(z)}{q(z)}=\rho A z^{\rho-1} L(z)+o\left(\frac{V(r)}{r}\right), \quad r \rightarrow \infty, z=r e^{i \varphi},|\varphi| \leq \alpha / 3, \\
\left(\frac{q^{\prime}(z)}{q(z)}\right)^{\prime}=\rho(\rho-1) A z^{\rho-2} L(z)+o\left(\frac{V(r)}{r^{2}}\right), \quad r \rightarrow \infty, z=r e^{i \varphi},|\varphi| \leq \alpha / 3, \\
\left(\frac{q^{\prime}(z)}{q(z)}\right)^{\prime}\left(\frac{q(z)}{q^{\prime}(z)}\right)^{2}=O\left(\frac{1}{V(r)}\right), \quad r \rightarrow \infty, z=r e^{i \varphi},|\varphi| \leq \alpha / 3 .
\end{gathered}
$$

It is clear from (14), (15) and (17) that the derivative $q^{\prime}(z)$ does not vanish in $\{z:|\arg z| \leq \alpha / 3,|z|>R\}$ for all large $R$.

To verify (13), we use induction. When $n=1,(13)$ is evident. Assume that (13) holds for some $n$. Then we have

$$
\left(\frac{d}{d z}\right)^{n+1} \frac{1}{q(z)}=\frac{d}{d z}\left[(-1)^{n}\left(\frac{q^{\prime}(z)}{q(z)}\right)^{n} \frac{1}{q(z)}\left(1+O\left(\frac{1}{V(r)}\right)\right)\right]
$$




$$
\begin{gathered}
=(-1)^{n}\left[n\left(\frac{q^{\prime}(z)}{q(z)}\right)^{n-1}\left(\frac{q^{\prime}(z)}{q(z)}\right)^{\prime} \frac{1}{q(z)}-\left(\frac{q^{\prime}(z)}{q(z)}\right)^{n+1} \frac{1}{q(z)}\right]\left(1+O\left(\frac{1}{V(r)}\right)\right) \\
+(-1)^{n}\left(\frac{q^{\prime}(z)}{q(z)}\right)^{n} \frac{1}{q(z)} \cdot O\left(\frac{1}{r V(r)}\right)=(-1)^{n+1}\left(\frac{q^{\prime}(z)}{q(z)}\right)^{n+1} \frac{1}{q(z)} \\
.\left\{\left[1-n\left(\frac{q^{\prime}(z)}{q(z)}\right)^{\prime}\left(\frac{q(z)}{q^{\prime}(z)}\right)^{2}\right]\left(1+O\left(\frac{1}{V(r)}\right)\right)-\left(\frac{q^{\prime}(z)}{q(z)}\right)^{-1} O\left(\frac{1}{r V(r)}\right)\right\} .
\end{gathered}
$$

This, together with (18) and (17), establishes (13).

\section{Proof of Theorem 4}

Suppose that $g$ is a function whose existence is established in Lemma 2. Let us show that there exists a real even entire function $G$ of exponential type with real zeros such that

$$
\lim _{|x| \rightarrow \infty \in \mathbf{R}} x^{n} G(x) g(x)=0, \quad n=1,2, \ldots
$$

Let $h$ be a function from Lemma 3 and let $m \geq 2+2 \pi / \sin (\pi \rho / 2)$ be an even number. Then, by (10) and (12), there is a constant $D>0$ such that for all real $x$,

$$
\log \left|h^{m}(x) g(x)\right| \leq-V(|x|)+D
$$

Set $G(z)=h^{m}(z)$. We see that an even stronger statement than (19) holds:

$$
|G(x) g(x)| \leq \exp \{-V(|x|)+D\} .
$$

Since $h$ is even, $h(i y)$ is real, so that $G$ is positive on $i \mathbf{R}$. Moreover, $G$ is bounded on $\mathbf{R}$ and all its zeros are real. A well-known result on entire functions of exponential type (see, e.g., [4], p. 240, Theorem 5) then implies the asymptotic equality

$$
\log G(i y)=A|y|+o(|y|), \quad|y| \rightarrow \infty
$$

where $A$ is some positive constant. In view of (10) and (11), this gives

$$
\begin{aligned}
\left|G\left( \pm i d_{k}\right) g\left( \pm i d_{k}\right)\right| & \rightarrow \infty, \quad k \rightarrow \infty, \\
\operatorname{sign}\left(G\left( \pm i d_{k}\right) g\left( \pm i d_{k}\right)\right) & =(-1)^{k}, \quad k=1,2, \ldots
\end{aligned}
$$

We see that there exists a small number $c>0$ that every interval $\left(c_{2 k-1}, c_{2 k}\right)$ contains at least two points, say $p_{2 k-1}$ and $p_{2 k}$, which are roots of the equation

$$
G(i y) g(i y)=-c .
$$


Set

$$
q(z)=\prod_{k=1}^{\infty}\left(1+\frac{z^{2}}{p_{k}^{2}}\right)
$$

and define

$$
f(z)=\frac{G(z) g(z)+c}{q(z)} .
$$

We claim that this function satisfies the conclusion of Theorem 4. Since $f$ is entire and is a ratio of two functions of exponential type, it is itself of exponential type.

Let us show that $f$ satisfies condition (3). Using (19), we get

$$
\begin{aligned}
& \log |f(x)|=\log \left|\frac{c+o(1)}{q(x)}\right|=\log \frac{1}{q(x)}+O(1) \\
& =\log \frac{1}{|g(x)|}+\log \left|\frac{g(x)}{q(x)}\right|+O(1), \quad|x| \rightarrow \infty .
\end{aligned}
$$

Since $c_{2 k-1}<p_{2 k-1}<p_{2 k}<c_{2 k}$, we see that

$$
\begin{gathered}
\log \left|\frac{g(x)}{q(x)}\right|=\sum_{k=1}^{\infty} \log \frac{1+x^{2} / c_{2 k-1}^{2}}{1+x^{2} / p_{2 k-1}^{2}}+\sum_{k=1}^{\infty} \log \frac{1+x^{2} / c_{2 k}^{2}}{1+x^{2} / p_{2 k}^{2}} \\
<\sum_{k=1}^{\infty} \log \frac{1+x^{2} / c_{2 k-1}^{2}}{1+x^{2} / p_{2 k-1}^{2}}=\log \left(1+\frac{x^{2}}{c_{1}^{2}}\right)+\sum_{k=1}^{\infty} \log \frac{1+x^{2} / c_{2 k+1}^{2}}{1+x^{2} / p_{2 k-1}^{2}}<\log \left(1+\frac{x^{2}}{c_{1}^{2}}\right) .
\end{gathered}
$$

One can get a similar estimate of $\log |g(x) / q(x)|$ from below. This gives

$$
\left.\log |f(x)|=\log \frac{1}{|g(x)|}+O(\log |x|)\right), \quad|x| \rightarrow \infty .
$$

Now (3) follows from (10).

It remains to verify that $f$ is non-oscillating, that is every derivative of $f$ has only a finite number of real zeros. Set $F(z)=G(z) g(z)$. It follows from (20) that

$$
\left(\frac{d}{d z}\right)^{n} f(z)=c\left(\frac{d}{d z}\right)^{n} \frac{1}{q(z)}+\left(\frac{d}{d z}\right)^{n} \frac{F(z)}{q(z)} .
$$

By construction, function $q$ is an entire function with purely imaginary roots at $\pm i p_{k}$. Denote by $n_{q}^{ \pm}(r)$ and $n_{g}^{ \pm}(r)$ the number of roots of $q$ and $g$ in $\{z:|z| \leq r, \pm \Im z>0\}$, respectively. It follows from (9) and the construction of $q$ that

$$
\left|n_{q}^{ \pm}(r)-n_{g}^{ \pm}(r)\right|=O(1), \quad r \rightarrow \infty .
$$

Since $n_{g}^{ \pm}(r)=V(r)(1+o(1)), n_{q}^{ \pm}(r)$ has the same asymptotics. It follows that $q$ is an entire function of completely regular growth with respect to the proximate 
order $\rho(r)$. Observe that $|q(z)| \leq q( \pm|z|)$, so that $q$ satisfies the assumption (ii) of Lemma 4. Thus, formula (13) holds. By Lemma 4, $q$ ' has only a finite number of real zeros; therefore, the first term on the right-hand side of (21) has only a finite number of real zeros. By (13) and (20), to finish the proof it now suffices to establish that

$$
\left(\frac{d}{d x}\right)^{n} \frac{F(x)}{q(x)}=o\left(\left|\frac{q^{\prime}(x)}{q(x)}\right|^{n} \frac{1}{q(x)}\right), \quad|x| \rightarrow \infty .
$$

We use Lemma 4 to get

$$
\begin{gathered}
\left(\frac{d}{d x}\right)^{n} \frac{F(x)}{q(x)}=\sum_{j=0}^{n} C_{n}^{j} F^{(j)}(x)\left(\frac{d}{d x}\right)^{n-j} \frac{1}{q(x)} \\
=\sum_{j=0}^{n} C_{n}^{j} F^{(j)}(x)\left[(-1)^{n-j}\left(\frac{q^{\prime}(x)}{q(x)}\right)^{n-j} \frac{1}{q(x)}\left(1+O\left(\frac{1}{V(|x|)}\right)\right)\right] \\
=\left(\frac{q^{\prime}(x)}{q(x)}\right)^{n} \frac{1}{q(x)} \sum_{j=1}^{n} C_{n}^{j} F^{(j)}(x)\left[(-1)^{n-j}\left(\frac{q(x)}{q^{\prime}(x)}\right)^{j}\left(1+O\left(\frac{1}{V(|x|)}\right)\right)\right], \\
|x| \rightarrow \infty .
\end{gathered}
$$

It follows from (17) and (14) that

$$
\frac{q(x)}{q^{\prime}(x)}=O\left(\frac{|x|}{V(|x|)}\right)=o(|x|), \quad|x| \rightarrow \infty .
$$

Since $F$ is an entire function of exponential type and $F(x)=O\left(|x|^{-m}\right)$ for any natural number $m$,

$$
F^{(j)}(x)=O\left(|x|^{-m}\right), \quad|x| \rightarrow \infty
$$

for any natural numbers $j$ and $m$. This establishes (22) and completes the proof of Theorem 4.

\section{Proof of Theorem 5 (i)}

Let $h, h(0)=1$, be a real entire function of exponential type whose existence is established by Lemma 3. We shall need the estimate.

$$
|h(x+i y)| \leq c \exp [a|y|-b V(|x|)], \quad|y| \leq|x|,
$$

where $a, b, c$ are positive constants.

Let $a$ be any number strictly greater than the type of $h$, and $L$ be a function analytic in $\{z:|\arg z|<\pi\}$ satisfying (14) and (15). Set

$$
h_{1}(z)=h(z) \exp \left[i a z+(1 / 2) z^{\rho} L(z)\right] .
$$


Clearly, $h_{1}$ is analytic in the quadrant $Q=\{z: 0 \leq \arg z \leq \pi / 2\}$. Formulas (14) and (12) show that $h_{1}$ is bounded on the positive half-axis. Further, it follows from (14), (15) and (2) that

$$
\Re\left(z^{\rho} L(z)\right)=r^{\rho} \cos (\rho \varphi) \cdot V(r)(1+o(1))=o(r), \quad r \rightarrow \infty .
$$

We see that $h_{1}$ is of order $\leq 1$ in $Q$ and bounded on the positive imaginary half-axis. By the Phragmén-Lindelöf principle, $h_{1}$ is bounded in $Q$. Hence

$$
|h(z)| \leq c\left|\exp \left[-i a z-(1 / 2) z^{\rho} L(z)\right]\right|, \quad z \in Q
$$

$c$ being a positive constant. Recalling that (by (2)) $\rho \leq 1$ and using (24), we obtain (23) for the angle $\{z=x+i y: 0 \leq y \leq x\}$. For the other three angles of the form $\{z: 0 \leq \pm y \leq \pm x\}$, the proof of (23) is similar.

Set

$$
f(z)=\frac{1-h(z)}{z} .
$$

We show that (4) holds for this function (and hence it is a non-oscillating PWfunction). Clearly,

$$
f^{(n)}(x)=\frac{(-1)^{n} n !}{x^{n+1}}+\left(\frac{h(x)}{x}\right)^{(n)} .
$$

We estimate the second term in the right-hand side for large $x$. Let

$$
M_{x}(r)=\max _{|z-x|=r}|h(z)| .
$$

Inequality (23) shows that for $x>r$,

$$
M_{x}(r) \leq c \exp \{a r-b V(x-r)\}
$$

The Cauchy integral formula gives

$$
\left|\left(\frac{h(x)}{x}\right)^{(n)}\right| \leq n ! \frac{M_{x}(r)}{(x-r) r^{n}} .
$$

It is now clear that $f^{(n)}(x)$ does not vanish, provided that

$$
\left|\left(\frac{h(x)}{x}\right)^{(n)}\right| \leq \frac{n !}{x^{n+1}} .
$$

Hence, $f^{(n)}(x)$ does not vanish if

$$
n ! \frac{M_{x}(r)}{(x-r) r^{n}} \leq \frac{n !}{x^{n+1}}
$$


For $r<x / 2$, this can be rewritten as

$$
M_{x}(r) \leq \frac{1}{2}\left(\frac{r}{x}\right)^{n}
$$

By (25), the last inequality holds if

$$
c \exp \{a r-b V(x-r)\} \leq \frac{1}{2}\left(\frac{r}{x}\right)^{n} .
$$

Observe that there is a constant $q>0$ such that

$$
V(x-r) \geq V(x / 2) \geq q V(x), \quad 0<x<r / 2 .
$$

The first inequality holds because $V$ is increasing; the second follows from a wellknown property of proximate order ([2], p. 73; [4], p. 33). Therefore, (26) holds if

$$
a r-b q V(x) \leq n \log (r / x)+c_{1},
$$

where $c_{1}$ is a positive constant. Set

$$
r=\frac{b q}{2 a} V(x)
$$

Then we see that $f^{(n)}(x)$ cannot vanish provided that

$$
-\frac{b q}{2} V(x) \leq n \log \left(\frac{b q}{2 a} \cdot \frac{V(x)}{x}\right)+c_{1}
$$

or

$$
V(x) \geq \frac{2 n}{b q} \log \left(\frac{2 a}{b q} \cdot \frac{x}{V(x)}\right)-\frac{2 c_{1}}{b q} .
$$

Since $V(x) \geq \delta x^{\rho / 2}$ for all $x \geq 1$ if $\delta$ is sufficiently small, $v(u) \leq(u / \delta)^{2 / \rho}$ for all sufficiently large $u$. Taking this into account, one easily checks that $x=v(C n \log n)$ satisfies (27) for $n=1,2, \ldots$, provided that $C$ is large enough. Hence (4) holds.

\section{Proof of Theorem 5 (ii) and Corollary 1}

For any natural number $n$, we denote by $\nu_{n}(t)$ the number of zeros of $f^{(n)}$ in the disc $\{z:|z| \leq t\}$ (counting multiplicities). If $f^{(n)}(0) \neq 0$, then by Jensen's formula,

$$
\int_{0}^{r} \frac{\nu_{n}(t)}{t} d t=\frac{1}{2 \pi} \int_{-\pi}^{\pi} \log \left|f^{(n)}\left(r e^{i \varphi}\right)\right| d \varphi-\log \left|f^{(n)}(0)\right|, \quad r \geq 0 .
$$

We choose $r=\operatorname{er}(n, f)$ and estimate the left-hand (right-hand) side of (28) from below (above) for this value of $r$. 
Observe that, by the definition of $r(n, f)$ and $p(n, f)$, we have

$$
\nu_{n}(r(n, f)) \geq p(n, f)
$$

Since

$$
\int_{0}^{e r} \frac{\nu_{n}(t)}{t} d t \geq \int_{r}^{e r} \frac{\nu_{n}(t)}{t} d t \geq \nu_{n}(r)
$$

we get

$$
\int_{0}^{e r(n, f)} \frac{\nu_{n}(t)}{t} d t \geq p(n, f)
$$

To estimate the first term of the right-hand side of (28), we observe that $f$ admits the representation

$$
f(z)=\int_{-1}^{1} e^{i z t} \psi(t) d t
$$

where $\psi \in L_{2}(-1,1)$; therefore,

$$
\begin{gathered}
\left|f^{(n)}(z)\right|=\left|\int_{-1}^{1} e^{i z t}(i t)^{n} \psi(t) d t\right| \\
\leq \int_{-1}^{1} e^{-y t}|t|^{n}|\psi(t)| d t \leq \int_{-1}^{1} e^{-y t}|\psi(t)| d t \leq\|\psi\|_{2} e^{|y|}, \quad n=0,1,2, \ldots
\end{gathered}
$$

Hence

$$
\frac{1}{2 \pi} \int_{-\pi}^{\pi} \log \left|f^{(n)}\left(r e^{i \varphi}\right)\right| d \varphi \leq \frac{1}{2 \pi} \int_{-\pi}^{\pi} \log \left(\|\psi\|_{2} e^{r|\sin \varphi|}\right) d \varphi=\frac{2}{\pi} r+\log \|\psi\|_{2}
$$

and

$$
\frac{1}{2 \pi} \int_{-\pi}^{\pi} \log \left|f^{(n)}\left(e r(n, f) e^{i \varphi}\right)\right| d \varphi \leq \frac{2}{\pi} \operatorname{er}(n, f)+\log \|\psi\|_{2} .
$$

To estimate the second term of the right-hand side of (28), we need the following lemma, whose proof will be given in the next section of the paper.

Lemma 5. Let $f$ be an entire function admitting the representation

$$
f(z)=\int_{-1}^{1} e^{i z t} d \mu(t)
$$

where $\mu$ is a complex-valued Borel measure on $[-1,1]$ of finite total variation such that $\{-1,1\} \subset \operatorname{supp} \mu$. Then there exists an increasing sequence $\left\{n_{j}\right\}_{j=1}^{\infty}$ of natural numbers such that

$$
\text { (i) } \lim _{j \rightarrow \infty} n_{j+1} / n_{j}=1
$$

(ii) $\log \left|f^{(n)}(0)\right|=o(n), \quad n=n_{j} \rightarrow \infty$. 
By Lemma 5, we have

$$
\log \left|f^{(n)}(0)\right|=o(n), \quad n=n_{j} \rightarrow \infty .
$$

Substituting this, (29) and (30) into (28), we obtain

$$
p(n, f) \leq \frac{2}{\pi} \operatorname{er}(n, f)+o(n), \quad n=n_{j} \rightarrow \infty .
$$

By Rolle's theorem, $p(n, f) \geq n$; therefore, (32) yields

$$
r(n, f) \geq \frac{\pi}{2 e} n+o(n), \quad n=n_{j} \rightarrow \infty .
$$

Taking into account that $r(n, f)$ increases in $n$ and the sequence $\left\{n_{j}\right\}_{j=1}^{\infty}$ satisfies Lemma 5(i), we get (5).

Let $f$ be the function whose existence has been established in Theorem 5 (i). Evidently, (32) is applicable to $f$. Using the inequality (4), we get

$$
p(n, f) \leq \frac{2}{\pi} e v(C n \log n)+o(n), \quad n=n_{j} \rightarrow \infty .
$$

Since $p(n, f)$ increases in $n$ and the sequence $\left\{n_{j}\right\}_{j=1}^{\infty}$ satisfies Lemma 5(i), we can increase the constant $C$ in such a way that (6) will hold.

\section{Proof of Lemma 5}

Note that the equality

$$
\limsup _{n \rightarrow \infty} \frac{1}{n} \log \left|f^{(n)}(0)\right|=0
$$

is trivially true because $f$ is of exponential type 1. Therefore, the existence of some sequence $\left\{n_{j}\right\}_{j=1}^{\infty}$ satisfying (ii) is evident. We show that such a sequence can be chosen to be rather dense, namely that (i) is fulfilled. First we prove this under the additional assumption that the measure $\mu$ is even, that is the function $f$ admits the representation

$$
f(z)=\int_{0}^{1} \cos (z t) d \mu(t)
$$

where $1 \in \operatorname{supp} \mu$. In this case,

$$
f^{(2 k)}(0)=(-1)^{k} \int_{0}^{1} t^{2 k} d \mu(t), \quad f^{(2 k+1)}(0)=0, \quad k=0,1, \ldots
$$

We prove the lemma by contradiction. Let us assume that Lemma 5 is wrong. Then there exist numbers $q>1, \varepsilon>0$ and a sequence of disjoint intervals $\left[a_{j}, b_{j}\right]$ such that $a_{j}$ and $b_{j}$ are natural numbers satisfying the condition

$$
b_{j} / a_{j} \geq q, \quad j=0,1,2, \ldots
$$




$$
\limsup _{k \rightarrow \infty, k \in A} \frac{1}{k} \log \left|f^{(2 k)}(0)\right|<-\varepsilon
$$

where

$$
A=\bigcup_{j=1}^{\infty}\left[a_{j}, b_{j}\right]
$$

Consider the function

$$
F(z)=\int_{0}^{1} t^{2 z+2} d \mu(t) .
$$

This function is analytic and bounded in the closed half-plane $\{z: \Re z \geq 0\}$. Since $F(k-1)=(-1)^{k} f^{(2 k)}(0), k=0,1, \ldots,(36)$ implies

$$
\limsup _{k \rightarrow \infty} \frac{1}{k} \log |F(k)|=0 .
$$

Hence, since $F$ is bounded on $\mathbf{R}$, we have

$$
\limsup _{x \rightarrow+\infty} \frac{1}{x} \log |F(x)|=0 .
$$

On the other hand, (36) implies

$$
\limsup _{k \rightarrow \infty, k \in A} \frac{1}{k} \log |F(k)|<-\varepsilon .
$$

By a well-known result ([5], p. 104, Theorem 3),

$$
\begin{aligned}
\log |F(z)| & =-\frac{x}{\pi} \int_{-\infty}^{\infty} \frac{d \nu(s)}{x^{2}+(y-s)^{2}}+\sum_{l} \log \left|\frac{z-a_{l}}{z+\bar{a}_{l}}\right|-c x \\
& =:-u_{1}(z)+u_{2}(z)-c x
\end{aligned}
$$

where $\nu$ is a non-negative Borel measure on $\mathbf{R}$ such that

$$
\int_{-\infty}^{\infty} \frac{d \nu(s)}{1+s^{2}}<\infty
$$

the $a_{l}$ 's are points in the half-plane $\{z: \Re z>0\}$ satisfying the condition

$$
\sum_{l} \frac{\cos \left(\arg a_{l}\right)}{\left|a_{l}\right|}<\infty
$$

and $c$ is a non-negative constant.

Observe that (37) yields $c=0$, and (40) implies $u_{1}(x)=o(x), \quad x \rightarrow+\infty$. Therefore, by (38) we see that

$$
\limsup _{k \rightarrow \infty} \frac{u_{2}(k)}{k}<-\varepsilon
$$


To prove the lemma, we show that this inequality cannot hold.

By the Hayman-Azarin theorem (see, e.g., [5], p. 109, Theorem 1),

$$
u_{2}(z) \geq-(1 / 2) \varepsilon|z|, \quad \Re z>0,
$$

outside some exceptional set of discs $C_{m}=\left\{z:\left|z-z_{m}\right|<\delta_{m}\right\}$ of finite view, that is, such that

$$
\sum_{m}\left(\delta_{m} /\left|z_{m}\right|\right)<\infty
$$

Since

$$
\sum_{\left|z_{m}\right|>R}\left(\delta_{m} /\left|z_{m}\right|\right) \rightarrow 0 \quad \text { as } R \rightarrow+\infty,
$$

we can choose $R$ and the rays $\Lambda_{ \pm}:=\{z: \arg z= \pm \theta\}, 0<\theta<\pi / 4$, in such a way that

$$
\left(\Lambda_{ \pm} \cap\{z:|z|>R\}\right) \cap \bigcup_{m} C_{m}=\emptyset .
$$

The Hayman-Azarin theorem ([5], p. 109) also implies that there is a sequence of segments

$$
\left\{\left[d_{p}, d_{p}+\eta_{p}\right]\right\}_{p=1}^{\infty}, \quad 0<d_{p}<d_{p}+\eta_{p}<d_{p+1} \uparrow+\infty,
$$

satisfying the condition

$$
\sum_{p=1}^{\infty}\left(\eta_{p} / d_{p}\right)<\infty
$$

and such that (43) holds outside the half-annuli

$$
\left\{z: d_{p}<|z|<d_{p}+\eta_{p}, \Re z>0\right\} .
$$

Let us consider the system of sectors

$$
K_{p}:=\left\{z: d_{p}<|z|<d_{p}+\eta_{p},|\arg z|<\theta\right\}, \quad p \geq p_{0},
$$

where $p_{0}$ is so large that the circumference $\{z:|z|=R\}$ does not intersect $K_{p}$ for $p \geq p_{0}$. Evidently, the sectors $K_{p}$ are pairwise disjoint and their union covers the set $A \cap\{z:|z|>R\}$. Moreover, we have

$$
u_{2}(z) \geq-(\varepsilon / 2)|z|, \quad z \in \partial K_{p}, p \geq p_{0} .
$$

We split the system of sectors $\left\{K_{p}\right\}_{p=p_{0}}^{\infty}$ into two groups. The first (second) one consists of those, for which $\eta_{p} \geq 1\left(\eta_{p}<1\right)$. We denote the sectors of the first (second) group by $K_{p}^{\prime}\left(K_{p}^{\prime \prime}\right)$.

Setting

$$
A^{\prime}=A \cap \bigcup_{p} K_{p}^{\prime}
$$


we obtain

$$
\sum_{k \in A^{\prime}} \frac{1}{k} \leq \sum_{\eta_{p} \geq 1} \sum_{d_{p} \leq k \leq d_{p}+\eta_{p}} \frac{1}{k} \leq \sum_{\eta_{p} \geq 1} \frac{\eta_{p}+1}{d_{p}} \leq \sum_{\eta_{p} \geq 1} \frac{2 \eta_{p}}{d_{p}} \leq 2 \sum_{p=1}^{\infty} \frac{\eta_{p}}{d_{p}}<\infty .
$$

Since (33) implies

$$
\sum_{k \in A} \frac{1}{k}=\infty
$$

using the notation

$$
A^{\prime \prime}=A \cap \bigcup_{p} K_{p}^{\prime \prime}
$$

we have

$$
\sum_{k \in A^{\prime \prime}} \frac{1}{k}=\infty
$$

Each point $k \in A^{\prime \prime}$ belongs at least to one sector $K_{p}^{\prime \prime}$. Moreover, each sector $K_{p}^{\prime \prime}$ contains at most one point from $A^{\prime \prime}$ because $\eta_{p}<1$. Thus, to each $k \in A^{\prime \prime}$, there corresponds a unique sector $K_{p}^{\prime \prime}$, which will be denoted by $K_{p(k)}^{\prime \prime}$.

Now observe that each sector $K_{p(k)}^{\prime \prime}$ must contain at least one point $a_{l}$ from the representation (39). Indeed, otherwise, the function $u_{2}$ would be harmonic in $K_{p(k)}^{\prime \prime}$, and so

$$
u_{2}(k) \geq \min _{z \in \partial K_{p(k)}^{\prime \prime}} u_{2}(z) \geq-(\varepsilon / 2)\left(k+\eta_{p(k)}\right) \geq-(\varepsilon / 2)(k+1) .
$$

This contradicts condition (42) for sufficiently large $k$.

Thus, to each sufficiently large $k \in A^{\prime \prime}$, there corresponds at least one point $a_{l}=a_{l(k)} \in K_{p(k)}^{\prime \prime}$; and to different values of $k$, there correspond different values $a_{l(k)}$. Since both $k$ and $a_{l(k)}$ belong to the same $K_{p(k)}^{\prime \prime}$,

$$
|k-| a_{l(k)}||<\eta_{p(k)}<1
$$

Therefore, (44) implies

$$
\sum_{k \in A^{\prime \prime}} \frac{1}{\left|a_{l(k)}\right|}=\infty
$$

On the other hand,

$$
\sum_{k \in A^{\prime \prime}} \frac{1}{\left|a_{l(k)}\right|} \leq \frac{1}{\cos (\pi / 4)} \sum_{k \in A^{\prime \prime}} \frac{\cos \left(\arg a_{l(k)}\right)}{\left|a_{l(k)}\right|} \leq \frac{1}{\cos (\pi / 4)} \sum_{l} \frac{\cos \left(\arg a_{l}\right)}{\left|a_{l}\right|} .
$$

Clearly, this inequality and (41) contradict each other, which proves Lemma 5 under the additional assumption that the function $f$ admits the representation (34) and $1 \in \operatorname{supp} \mu$. 
If $f$ admits the representation

$$
f(z)=\int_{0}^{1} \sin (t z) d \mu(t), \quad 1 \in \operatorname{supp} \mu,
$$

then the proof is similar. In general, when $f$ satisfies (31), we set

$$
f_{1}(z)=\int_{0}^{1} \cos (t z) d \mu_{1}(t), \quad f_{2}(z)=\int_{0}^{1} \sin (t z) d \mu_{2}(t),
$$

where

$$
\mu_{1}(E)=\mu(E)+\mu(-E), \quad i \mu_{2}(E)=\mu(E)-\mu(-E)
$$

and observe that $1 \in \operatorname{supp} \mu_{1} \bigcup \operatorname{supp} \mu_{2}$. Therefore, we can apply what we have already proved to either $f_{1}$ or $f_{2}$. Noting that

$$
f^{(2 k)}(0)=f_{1}^{(2 k)}(0), \quad f^{(2 k+1)}(0)=f_{2}^{(2 k+1)}(0), \quad k=0,1,2, \ldots,
$$

we obtain the desired assertion.

\section{Proof of Theorem 2}

We begin this section with the following theorem, which we think has independent interest.

Theorem 6. Suppose $k \geq 0$ is an integer, $f$ is a real $P W$-function, and $F$ is its spectral function. Assume that the derivative $f^{(k+3)}(x)$ has only a finite number of changes of sign on the real line. Then $F \in C^{k}(\mathbf{R} \backslash\{0\})$.

Theorem 2 follows immediately from this result and the definition of a nonoscillating PW-function.

Another immediate corollary of Theorem 6 is the following result, which for any $k \geq 0$ gives a description of a wide class of real PW-functions $f$ for which the $n$-th derivative of $f, n \geq 3$, must have infinitely many real zeros.

Corollary 3. Let $f$ be a real $P W$-function with spectral function $F$. Assume $F \notin C^{k}(\mathbf{R} \backslash\{0\})$ for some integer $k$. Then $f^{(k+3)}$ has infinitely many changes of sign.

We deduce Theorem 6 from

Lemma 6. Let $g \in L_{1}(\mathbf{R})$ and $g(x) \geq 0$ for all large $|x|$, and let $G$ be the spectral function of $g$. Suppose there is an even integer $n \geq 2$ such that $G$ is $n$ times differentiable at the origin. Then $G \in C^{n}(\mathbf{R})$. 
Proof. When $g$ is non-negative on $\mathbf{R}$, this lemma is contained in the wellknown Lévy theorem (see Theorem 2.1.1 and its Corollary 1 in [6], p. 21).

In the general case, there exists $a>0$ such that $g(x) \geq 0$ for $|x| \geq a$. Write

$$
g_{1}(x):=\chi_{(-a, a)}(x) g(x), \quad g_{2}(x):=g(x)-g_{1}(x),
$$

where $\chi_{(-a, a)}$ is the characteristic function of the interval $(-a, a)$. Then $G=G_{1}+G_{2}$, where $G_{j}$ is the spectral function of $g_{j}, j=1,2$. Clearly, $G_{1}$ can be continued to the complex plane as an entire function, so $G_{1}$ is infinitely differentiable. The lemma now follows from Corollary 1 in [6], according to which $G_{2}^{(n)}$ exists and is continuous on $\mathbf{R}$.

Proof of Theorem 6. Let $f$ be a real PW-function with spectral function $F$. We begin with the observation that if $f^{\prime}$ has only a finite number of real zeros, then $f^{\prime} \in L_{1}(\mathbf{R})$. Indeed, there is a number $a>0$ such that $f^{\prime}$ does not change the sign (i.e., is either non-positive or non-negative) in $(a, \infty)$ and in $(-\infty,-a)$. This gives

$$
\begin{aligned}
& \int_{-\infty}^{\infty}\left|f^{\prime}(x)\right| d x=\left|\int_{-\infty}^{-a} f^{\prime}(s) d s\right|+\int_{-a}^{a}\left|f^{\prime}(s)\right| d s+\left|\int_{a}^{\infty} f^{\prime}(s) d s\right| \\
& \leq|f(-a)|+\sqrt{2 a}\left\|f^{\prime}\right\|_{L_{2}}+|f(a)|<\infty .
\end{aligned}
$$

The spectral function of $f^{\prime}$ is $i s F(s)$. Since it is the inverse Fourier transform of $f^{\prime}$, which belongs to $L_{1}(\mathbf{R})$, it follows that $s F(s)$ is continuous on the real line. Hence, for any $n=1,2, \ldots$, the function $s^{n} F(s)$ is $n-1$ times differentiable at the origin.

In what follows, we assume that $f(x) \geq 0$ for all large negative $x$ (otherwise, consider the function $-f(x)$ ). Then $f$ satisfies one of the conditions

(i) $f(x) \geq 0$ for all large positive $x$,

(ii) $f(x) \leq 0$ for all large positive $x$.

(i) By Rolle's theorem, for any integer $s, 0 \leq s \leq k+2$, the derivative $f^{(s)}(x)$ has only a finite number of real zeros. It is then clear that for any even number $s$, $f^{(s)}(x) \geq 0$ for all large $|x|$.

Assume that $k$ is even. Then $f^{(k+2)}(x) \geq 0$ for all large $|x|$. The spectral function of $f^{(k+2)}$ is $(i s)^{k+2} F(s)$. It is $k+1$ times differentiable at the origin. Hence, by Lemma 6 with $g=f^{(k+2)}$, we conclude that $\left((i s)^{k+2} F(s)\right)^{(k)}$ exists and is continuous on $\mathbf{R}$. It follows that $F \in C^{k}(\mathbf{R} \backslash\{0\})$.

Assume now that $k$ is odd. Then $f^{(k+3)}(x) \geq 0$ for all large $x$. The same argument shows that the derivative $F \in C^{k+1}(\mathbf{R} \backslash\{0\})$. 
(ii) Clearly, for any odd integer $s, 1 \leq s \leq k+3$, we have $f^{(s)}(x) \geq 0$ for all large $|x|$. The same argument as in (i) establishes that $F \in C^{k}(\mathbf{R} \backslash\{0\})$ if $k$ is odd, and $F \in C^{k+1}(\mathbf{R} \backslash\{0\})$ if $k$ is even.

Remark. The observation at the beginning of the proof implies that, for any non-oscillating PW-function $f$, one has $f^{(k)} \in L_{1}(\mathbf{R}), k=1,2, \ldots$ For $k=0$, this is not always true, as the example of functions $f_{1}, f_{2}$ in Theorem 1 shows. It can be shown that $f^{(k)} \in L_{1}(\mathbf{R}), k=1,2, \ldots$ for any real PW-function $f$ such that $f^{\prime}$ has only finitely many real zeros.

Acknowledgement. A substantial part of this research was done during a visit of the second-named author to Bilkent University at Ankara. This visit was supported by the Scientific and Technical Research Council of Turkey (TÜBITAK).

\section{REFERENCES}

[1] J. Clunie, Q. I. Rahman and W. J. Walker, On entire functions of exponential type bounded on the real axis J. London Math. Soc. (2) 61 (2000), 163-176.

[2] A. A. Goldberg and I. V. Ostrovskii, Value Distribution of Meromorphic Functions, Nauka, Moscow, 1970 (Russian).

[3] J. R. Higgins, Sampling Theory in Fourier and Signal Analysis: Foundations, Clarendon Press, Oxford, 1996.

[4] B. Ya. Levin, Distribution of Zeros of Entire Functions, Translations of Mathematical Monographs, Vol. 5, Amer. Math. Soc., Providence, RI, 1980.

[5] B. Ya. Levin, Lectures on Entire Functions, Translations of Mathematical Monographs, Vol. 150. Amer. Math. Soc., Providence, RI, 1996.

[6] Ju. V. Linnik and I. V. Ostrovskii, Decomposition of Random Variables and Vectors, Translations of Mathematical Monographs, Vol. 48, Amer. Math. Soc., Providence, RI, 1977.

[7] I. V. Ostrovskii and A. Ulanovskii, Non-oscillating Paley-Wiener functions, C. R. Acad. Sci. Paris, Série 1333 (2001), 735-740.

[8] W. J. Walker, Oscillatory properties of Paley-Wiener functions, Indian J. Pure Appl. Math. 25 (1994), 1253-1258.

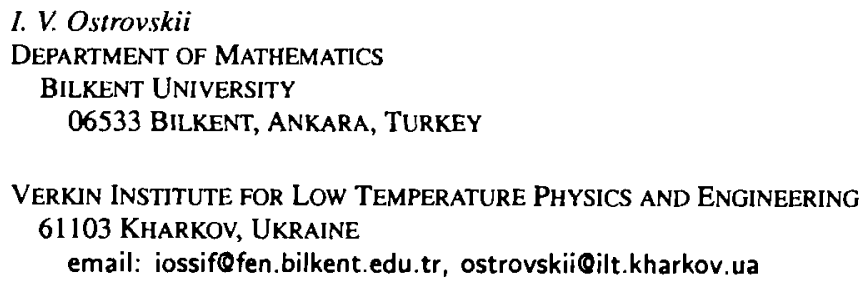

A. Ulanovskii

STAVANGER UNIVERSITY COLLEGE

P.O. BOX 2557 UlLANDHAUG 4091 STAVANGER, NORWAY email: Alexander.Ulanovskii@tn.his.no 